msh-mss Mathématiques et sciences humaines

152 | Hiver 2000

Varia

\title{
R. L. Graham, M. Grötschel, L. Lovasz (sous la direction de), "Handbook of combinatorics", Amsterdam, North-Holland, 1995 (2 volumes)
}

R. L. Graham, M. Grötschel, L. Lovasz (sous la direction de), "Handbook of combinatorics", Amsterdam, North-Holland, 1995 (2 volumes)

Olivier Hudry

\section{OpenEdition \\ Journals}

Édition électronique

URL : http://journals.openedition.org/msh/2869

DOI : $10.4000 / \mathrm{msh} .2869$

ISSN : $1950-6821$

Éditeur

Centre d'analyse et de mathématique sociales de l'EHESS

Édition imprimée

Date de publication : 1 décembre 2000

ISSN : 0987-6936

Référence électronique

Olivier Hudry, « R. L. Graham, M. Grötschel, L. Lovasz (sous la direction de), "Handbook of

combinatorics", Amsterdam, North-Holland, 1995 (2 volumes) », Mathématiques et sciences humaines

[En ligne], 152 | Hiver 2000, mis en ligne le 10 février 2006, consulté le 25 septembre 2020. URL

http://journals.openedition.org/msh/2869; DOI : https://doi.org/10.4000/msh.2869 


\section{BIBLIOGRAPHIE CRITIQUE}

R. L. GRAHAM, M. GRÖTSCHEL, L. LOVÁSZ (sous la direction de), Handbook of combinatorics, North-Holland, Amsterdam, 1995, deux volumes.

Voilà ce qui s'appelle une somme !... L'objectif déclaré, et de plus atteint, des deux gros volumes constituant ce Handbook of combinatorics est de fournir une bonne vue d'ensemble du vaste domaine de la combinatoire, en proposant une description des modèles et méthodes classiques rencontrés dans cette discipline, en recensant les résultats essentiels obtenus dans chaque secteur abordé et en essayant d'indiquer les tendances principales de la combinatoire.

Ambitieuse entreprise donc, qu'ont relevée R.!L.!Graham, M.!Grötschel et L.!Lovász en s'entourant d'un grand nombre de collaborateurs réputés. Il en résulte deux forts volumes totalisant plus de deux mille deux cents pages regroupées en cinq parties!:

- La première est consacrée aux structures combinatoires classiques : les graphes (six chapitres plus une annexe rédigés par J.!A.!Bondy, A.!Frank, M.!Karonski, W.!R.!Pulleyblank, P.!D.!Seymour, C.!Thomassen et B.!Toft), les ensembles et relations finis (deux chapitres, par P.!Duchet et W.!T.!Trotter), les matroïdes (trois chapitres, par R.!E.!Bixby, W.!H.!Cunningham, P.!D.!Seymour et D.!J.!A.!Welsh), les structures symétriques (cinq chapitres, par A.!E.!Brouwer, P.!J.!Cameron, W.!H.!Haemers et J.!H.!van Lint) et enfin les structures combinatoires en géométrie et en théorie des nombres (quatre chapitres, par P.!Erdös, V.!Klee, P.!Kleinschmidt, J.!C.!Lagarias, C.!Pomerance, G.!Purdy et A.!Sárközy). Cette première partie occupe le premier volume à elle seule, avec plus de mille pages.

- La deuxième partie s'intitule "Aspects", car elle entre dans le détail de certains aspects de la combinatoire, allant par exemple de la théorie de Ramsey (J.!Ne šetřil) et de l'analyse combinatoire (I.!M.!Gessel et R.!P.!Stanley) à la théorie de la complexité (D.!B.!Shmoys et É.!Tardos) en passant par l'optimisation combinatoire (M.!Grötschel et L.!Lovász) et cinq autres chapitres (L.!Babai, J.!Beck, B.!Bollobás, P.!Frankl, A.!M.!Odlyzko, V.!T.!Sós).

- Dans la troisième partie et en cinq chapitres sont décrites des méthodes et des outils fréquemment utilisés en combinatoire : la théorie polyédrale (A.!Schrijver), l'algèbre (N.!Alon) et particulièrement l'algèbre linéaire (C.!D.!Godsil avec une annexe de L.!Lovász), les méthodes probabilistes (J.!Spencer) ou topologiques (A.!Björner).

- Les applications habituelles de la combinatoire forment la quatrième partie, en sept chapitres : applications en recherche opérationnelle (A.!W.!J.!Kolen et J.!K.!Lenstra), en génie électrique (A.!Recski), en physique statistique (C.!D.!Godsil, M.!Grötschel et D.!J.!A.!Welsh), en chimie (D.!H.!Rouvray), en biologie moléculaire (M.!S.!Waterman), en informatique (L.!Lovász, D.!B.!Shmoys et É.!Tardos) et en mathématiques (L.!Lovász, L.!Pyber, D. J. A.!Welsh et G. (M..'Ziegler). 
- Enfin la conclusion, sous le titre "Horizons", donne un aperçu sur la combinatoire infinie (A.!Hajnal), sur les jeux combinatoires (R.!K.!Guy) et sur l'histoire de la combinatoire (N.!L.!Biggs, E.!K.!Lloyd et R.!J.!Wilson).

Ces cinq parties sont complétées par deux index très fournis : le premier concerne les auteurs cités (quarante-cinq pages sur deux colonnes), le second, les sujets par motsclés (quarante-quatre pages, sur deux colonnes aussi). Chaque chapitre possède sa propre bibliographie.

$\mathrm{Au}$ total, cinquante-trois auteurs se partagent donc quarante-quatre chapitres et deux petites annexes. La plupart des chapitres (trente-deux, pour être précis) ont entre trente et soixante pages, le plus long (un des six chapitres consacrés aux graphes) comptant cent huit pages et le plus court quatorze, pour une moyenne de quarante-neuf pages environ.

Cette simple énumération suffit à montrer l'immense étendue du domaine couvert!: le lecteur qui réussira à tout assimiler pourra se targuer d'un savoir encyclopédique en la matière!! Bien sûr, ces synthèses n'épuisent pas leurs sujets : on se doute bien que deux mille pages, et même beaucoup plus, ne permettent pas de décrire exhaustivement le monde riche et toujours en progression de la combinatoire (ce qui, du reste, n'était pas l'objectif des auteurs, comme on l'a vu plus haut). Par conséquent, il ne sera pas étonnant de trouver des points que le spécialiste jugera trop peu développés à son goût. De même, comme il s'agit pour certains chapitres d'une présentation synthétique de sujets classiques, le même spécialiste ne sera pas surpris de retrouver parfois une approche déjà développée (mais réactualisée!!) dans des monographies, au risque d'éprouver une impression de "déjà vu" pour quelques passages. Il n'en reste pas moins que cette œuvre monumentale constitue une mine de résultats et de références irremplaçable, et il est certain que tout lecteur intéressé par la combinatoire y trouvera grandement matière pour enrichir ses connaissances.

$\mathrm{Du}$ reste, je ne suis pas sûr que ce soit seulement dans l'originalité ou l'hexhaustivité dans tous les domaines relevant de la combinatoire qu'il convient de chercher la qualité essentielle de cet ouvrage. Pour autant que je puisse en juger à partir des chapitres que j'ai lus ou parcourus (car, avouons-le, je ne prétends pas avoir étudié les deux tomes in extenso!!), son intérêt se trouve dans le fait qu'y est proposé, de manière cohérente et structurée, un large panorama des thèmes, des résultats et des approches qui constituent la combinatoire. De ce fait, le lecteur pourra sortir de son champ d'expertise et étendre ses compétences en s'informant sur ce que font ses collègues et comment ils le font dans des domaines connexes au sien ou plus éloignés. Ce que proposent donc d'acquérir ces deux gros volumes, ce n'est pas le dernier état d'un secteur très pointu et limité de la combinatoire, mais plutôt une plus grande familiarité avec des techniques ou des résultats qu'un chercheur rencontre ou même manipule au cours de ses investigations sans les maîtriser pleinement parce qu'ils sortent de sa sphère de recherche stricto sensu. De ce fait, même vieux de quelques années, cet ouvrage reste un outil indispensable.

Et c'est sans doute cet aspect didactique qui vaut au livre son nom de "manuel", le démarquant ainsi des monographies de recherche que, d'ailleurs, il n'a pas pour vocation de remplacer mais plutôt de prolonger auprès d'un public plus vaste. Car, on l'aura compris, le public concerné, que j'imagine plutôt composé de chercheurs en 
combinatoire, confirmés ou débutants, est large!: l'ampleur des thèmes abordés et la qualité des synthèses constituant les chapitres font de ce livre un manuel de référence que tout chercheur pourra consulter avec profit pour élucider une question relevant de la combinatoire ou pour se familiariser avec une technique classiquement utilisée dans ce domaine. À cet égard, les coordonnateurs ont bien choisi l'ensemble de leurs collaborateurs : chercheurs connus et reconnus, leurs contributions (du moins celles que j'ai lues ou feuilletées) confirment leurs talents de pédagogues.

À ces qualités concernant le fond, on ajoutera encore celles relatives à la forme!: la présentation du texte, agréable et commode, qui facilite la lecture linéaire ou au contraire le repérage rapide du résultat cherché (on peut du reste penser qu'il s'agira là du mode d'utilisation le plus fréquent); des tables des matières détaillées propres à chaque chapitre ; une mise en page permettant d'identifier aisément les théorèmes, propositions ou autres corollaires (peut-être regrettera-t-on que certaines définitions ou certaines notations ne jouissent pas de la même mise en évidence ; il est vrai cependant que les index très fournis et la mise en italiques de certains termes dans le texte atténue considérablement cette critique)!; des conjectures qui viennent heureusement compléter l'état des connaissances en indiquant, en outre, des pistes de recherche intéressantes (mais non triviales!!) ; des bibliographies complètes ; etc.

Finalement, on ne peut trouver qu'un seul défaut majeur à cet ouvrage, défaut qui limitera sans doute, hélas, sa diffusion auprès des acquéreurs individuels : son prix, à l'image de la qualité du contenu, c'est-à-dire élevé ; il faut compter environ cent soixante dollars par volume. Et ça aussi, c'est ce qui s'appelle une somme, mais cela en vaut la peine!!...

\section{O. Hudry}

E.N.S.T. - Département informatique et réseaux 